\title{
Contribuições da Radiofrequência em flacidez genital feminina: Uma Revisão da Literatura
}

\author{
Talita Pereira Leal' ; Juliana Amorim Borba Santos ${ }^{2}$
}

\begin{abstract}
Resumo: A flacidez cutânea dos grandes lábios é uma das principais queixas das mulheres influenciando no desempenho sexual por causar constrangimento com a aparência. Dentre vários recursos afim de melhorar o aspecto da região dos grandes lábios, a radiofrequência (RF) tem prometido grande eficácia em tratamentos estéticos corporal e facial. É um procedimento não invasivo e indolor, que promove um aquecimento e consequentemente uma remodelação das fibras de colágeno e elastina. O presente estudo tem por objetivo averiguar as contribuições da radiofrequência em flacidez genital feminina através de uma revisão de literatura. Este estudo caracteriza-se como pesquisa de revisão integrativa de bibliografia de abordagem qualitativa de caráter exploratório. A pesquisa foi feita à partir de busca em artigos publicados em revistas anais e outros periódicos em base de dados confiáveis como Scielo, Lilacs, Bireme, PubMed, entre os anos de 2011 e 2018, logo após realizou-se a construção de um quadro descritivo dos artigos selecionados e feito discussão com embasamento científico. De acordo com vários estudos realizados com as mulheres, os autores descrevem que o tratamento com a Radiofrequência $(\mathrm{RF})$ apresenta resultados satisfatórios e trazem benefícios para flacidez genital. A radiofrequência para o tratamento da flacidez genital dos grandes lábios tem se mostrado eficaz.
\end{abstract}

Palavras-Chave: Flacidez genital feminina. Grandes lábios. Radiofrequência.

\section{Contributions of Radiofrequency in Female Genital Flacidity: A Review Of The Literature}

\begin{abstract}
The cutaneous flaccidity of the large lips is one of the main complaints of women influencing sexual performance because it causes embarrassment with appearance. Among several resources to improve the appearance of the large lips region, radiofrequency (RF) has promised great effectiveness in aesthetic treatments both body and face. It is a non-invasive and painless procedure, which promotes a heating and consequently a remodeling of collagen and elastin fibers. This study aims to investigate the contributions of radiofrequency to female genital flaccidity through a literature review. This study is characterized as an integrative review of a qualitative exploratory bibliography. The research was done from the search of articles published in anal journals and other periodicals in reliable databases such as Scielo, Lilacs, Bireme, PubMed, between the years of 2011 and 2018, soon after the construction of a descriptive table of the selected articles and made discussion with scientific basis. According to several studies with women, the authors describe that the treatment with Radiofrequency (RF) presents satisfactory results and bring benefits to genital flaccidity. Radiofrequency therapy for the treatment of genital flaccidity of large lips has been shown to be effective.
\end{abstract}

Keywords: Female genital flaccidity. Large lips. Radiofrequency.

Autor: Talita Pereira Leal

Qualificação: Esteticista e Cosmetologa

E-mail: talitaaleall@gmail.com

Orientador: Juliana Amorim Borba Santos

Qualificação:Fisioterapeuta, Especialista em dermatofuncional

E-mail: julianaamorim@fainor.com.br

\section{Introdução}


A epiderme, camada mais superficial da pele, possui o epitélio estratificado queratinizado apresentando a flacidez cutânea como fator determinante. Na derme encontra-se fibras de colágeno, que é a proteína mais abundante do corpo humano, responsável pela integridade e resistência dos tecidos. A derme é uma camada bem vascularizada e possui também glândulas sebáceas e nervos, e as fibras elásticas e de colágeno. A pele tem como principais funções: cobertura de toda superfície do corpo, proteção contra agentes, controle da temperatura corporal e sensibilidade (SILVA, 2010).

Todos nós estamos sujeitos ao envelhecimento com o avançar da idade, com isso ocorre várias alterações cutâneas, alterções que modificam o aspecto da mesma (PINTO; MEJIA 2012). A flacidez cutânea ocorre em ambos os sexos, sendo mais comum no sexo feminino por vários fatores, excesso de sol, parto normal, efeito sanfona e o envelhecimento natural, principalmente o hormonal e fatores extrínsecos e intrísecos que causam agressões ao organismo com danos as estruturas da pele, provocando enrugamento e envelhecimento precoce (GOMES, 2014 e VIEIRA, 2016).

A flacidez por sua vez, tem sido um dos fatores de influência na má qualidade de vida sexual da mulher, resultando na perda de sensação física durante o ato sexual, levando essa mulher a desenvolver um sentimento de baixa autoestima. Com o intuito de solucionar essas alterações, as mulheres que sofrem com a frouxidão vaginal têm buscado por cirurgias plásticas genitais (NOGUEIRA, 2017).

Os grandes lábios vulvares sofrem alterações em seu tecido com o avançar da idade e por ser composto também por um tecido queratinizado e estratificado, não está isento da flacidez nessa região. Fatores como genéticos, hormonais e excesso de peso, influenciam no processo de envelhecimento cutâneo nessa região (JUNQUEIRA, 1995).

A radiofrequência tem se mostrado como um tratamento inovador que promete melhorar a aparência principalmente da flacidez cutânea dos grandes lábios. A radiofrequência é uma proposta não invasiva e indolor, atualmente muito procurada por representar modificações nas características estéticas (BRASIL, 2013).

Esse procedimento, atua nas camadas da pele promovendo uma vasodilatação, nutrição do tecido e estimula a síntese de colágeno e elastina. Devido a sua corrente de alta frequência, gera calor por conversão e efeito térmico no tecido (ZEMUNER et al., 2011 e BOCK et al., 2013). 
Como questão norteadora este trabalho faz o seguinte questionamento: Quais as contribuições da radiofrequência na flacidez genital feminina? O presente estudo tem por objetivo geral averiguar as contribuições da radiofrequência em flacidez genital feminina através de uma revisão de literatura.

\section{Metodologia}

Este estudo caracteriza-se como pesquisa de revisão integrativa de bibliografia de abordagem qualitativa de caráter exploratório.

A pesquisa bibliográfica é elaborada a partir de material já efetuado, constituído principalmente, de livros e artigos científicos e é importante para o levantamento de informações básicas sobre os aspectos direto e indiretamente ligados à temática (VERGARA, 2000).

A abordagem qualitativa apresenta os resultados através de percepções e análises. Ela descreve a complexidade do problema e a interação de variáveis (MORETTI, 2018).

As pesquisas exploratórias são úteis para determinar situações, averiguar alternativas ou expor novas ideias. Esses trabalhos são dirigidos durante o estágio inicial de um processo de pesquisa mais amplo, em que se procura explicar e definir a natureza de um problema e gerar mais informações que possam ser adquiridas para a realização de futuras pesquisas conclusivas (ZIKMUND, 2000).

As estratégias utilizadas pela pesquisa exploratória são vastas e variáveis. Os métodos empregados contêm: pesquisas em fontes secundárias, levantamentos de experiências, estudos de casos selecionados e observação informal (MATTAR, 2001).

A pesquisa foi feita à partir de busca em artigos publicados em revistas anais e outros periódicos em base de dados confiáveis como Scielo, Lilacs, Bireme, PubMed, entre os anos de 2011 e 2018, logo após foram encontrados artigos e destes foram utilizados para a construção de um quadro descritivo e discussão dos resultados com embasamento científico.

\section{Discussão dos Resultados}




\begin{tabular}{|c|c|c|c|c|}
\hline $\mathbf{N}^{\mathbf{o}}$ & AUTOR & ANO & TÍTULO & RESULTADOS \\
\hline 01 & Goodman et al. & 2011 & $\begin{array}{l}\text { The Sexual, } \\
\text { Psychological, } \\
\text { and Body Image } \\
\text { Health of Women } \\
\text { Undergoing } \\
\text { Elective } \\
\text { Vulvovaginal } \\
\text { Plastic/ Cosmetic } \\
\text { Procedures: A } \\
\text { Pilot Study }\end{array}$ & $\begin{array}{l}\text { A cirurgia vulvovaginal } \\
\text { (VVA) apresenta } \\
\text { aumento na excitação e } \\
\text { satisfação sexual após a } \\
\text { cirurgia. Em relação a } \\
\text { função sexual a cirurgia } \\
\text { vulvovaginal e a RF } \\
\text { alcançaram resultados } \\
\text { parecidos. }\end{array}$ \\
\hline 02 & Santos & 2014 & $\begin{array}{l}\text { Função Sexual } \\
\text { Após o } \\
\text { Tratamento com a } \\
\text { Radiofrequência } \\
\text { em Região } \\
\text { Genital Feminina: } \\
\text { Ensaio Clínico } \\
\text { Randomizado }\end{array}$ & $\begin{array}{l}\text { A RF mostra-se eficaz } \\
\text { para o tratamento da } \\
\text { flacidez cutânea dos } \\
\text { grandes lábios, pois } \\
\text { proporciona um avanço } \\
\text { significativo na excitação } \\
\text { e satisfação sexual das } \\
\text { mulheres. }\end{array}$ \\
\hline 03 & Leal & 2014 & $\begin{array}{l}\text { Radiofrequência } \\
\text { em Região } \\
\text { Genital Feminina: } \\
\text { um Ensaio } \\
\text { Clínico } \\
\text { Randomizado }\end{array}$ & $\begin{array}{l}\text { Em relação ao tratamento } \\
\text { com a RF os pacientes do } \\
\text { grupo estudo e do grupo } \\
\text { controle, ambos } \\
\text { relataram satisfação com } \\
\text { o resultado. }\end{array}$ \\
\hline 04 & Fistonic et al. & 2016 & $\begin{array}{c}\text { Short Time } \\
\text { Efficacy and } \\
\text { Safety of Focused } \\
\text { Monopolar } \\
\text { Radiofrequency } \\
\text { Device for Labial } \\
\text { Laxity } \\
\text { Improvement- } \\
\text { Noninvasive } \\
\text { Labia Tissue } \\
\text { Tightening. A } \\
\text { Prospective } \\
\text { Cohort Study }\end{array}$ & $\begin{array}{l}\text { Após o procedimento da } \\
\text { radiofrequência que é um } \\
\text { método não invasivo, foi } \\
\text { observado que a lassidão } \\
\text { labial teve uma melhora } \\
\text { e a pontuação total do } \\
\text { FSFI aumentou. }\end{array}$ \\
\hline 05 & Lordêlo et al. & 2016 & $\begin{array}{l}\text { Radiofrequency in } \\
\text { female external } \\
\text { genital cosmetics } \\
\text { and sexual } \\
\text { function: a } \\
\text { randomized } \\
\text { clinical trial }\end{array}$ & $\begin{array}{c}\text { Os profissionais } \\
\text { observaram um avanço } \\
\text { ao grupo tratado com RF, } \\
\text { sendo que a pontuação } \\
\text { geral da função sexual do } \\
\text { FSFI aumentou. }\end{array}$ \\
\hline 06 & Nogueira & 2017 & $\begin{array}{l}\text { Aplicação da } \\
\text { Radiofrequência } \\
\text { em Órgão Genital } \\
\text { Feminino: Um } \\
\text { Procedimento não }\end{array}$ & $\begin{array}{l}\text { Os estudos apresentaram } \\
\text { eficácia ao tratamento } \\
\text { com a radiofrequência, } \\
\text { com relação a flacidez } \\
\text { vaginal, diminuiu a }\end{array}$ \\
\hline
\end{tabular}


Invasivo no

Tratamento da

Flacidez de Pele Vulvar

07

Qureshi et al.

2017

08

Lalji e Lozanova

2017

09

Hashim et al.

2018

Frota et al.

2018
Nonsurgical

Vulvovaginal

Rejuvenation With

Radiofrequency and Laser

Devices:

A Literature

Review and

Comprehensive

Update for

Aesthetic

Surgeons

Evaluation of the safety and efficacy of a monopolar nonablative radiofrequency device for the improvement of vulvo-vaginal laxity and urinary incontinence

Noninvasive Vaginal Rejuvenation
10 elasticidade da vagina, melhorando a aparência e lubrificação vaginal.

A radiofrequência e aparelhos a laser melhoram a frouxidão vaginal e a síndrome geniturinária da menopausa. A RF é um método testado e aprovado por mulheres em vários estudos.

A RF é um método não invasivo e estimula as fibras de colágeno e elastina na vagina, tendo como resultado a melhora da frouxidão vulvo-vaginal e incontinência urinária. Este é um procedimento eficaz e seguro.

O laser de $\mathrm{CO}_{2} \mathrm{e} \mathrm{a}$ radiofrequência tem se mostrado eficaz em estudos para alcançar o rejuvenescimento vaginal não invasivo e reverter os efeitos do

envelhecimento genital feminino.

Tratamento com laser e radiofrequência da atrofia vulvovaginal: estudo bibliográfico
Lasers à base de $\mathrm{CO} 2$ e a radiofrequência apresentam resultados satisfatórios no tratamento da atrofia vulvovaginal, com melhorias nas atividades sexuais.

Fonte: Pesquisa dos autores

Existem vários métodos elétricos e mecânicos para o tratamento da flacidez tissular dos grandes lábios, durante muito tempo foi a cirúrgia plástica, como a lipoenxertia. Com o aumento da demanda em melhorar a aparência, foram desenvolvidos outros métodos não 
invasivos com a proposta de rejuvenescer a área vaginal e com recuperação rápida e sem riscos. Uma nova proposta de tratamento foi descoberta, a radiofrequência que é muito utilizado em tratamentos faciais e tem como objetivo também a remodelação de colágeno existente e formação de novas fibras de colágeno e elastina, sem agredir a epiderme.

\section{Figura 1 - Aparelho de Radiofrequência}

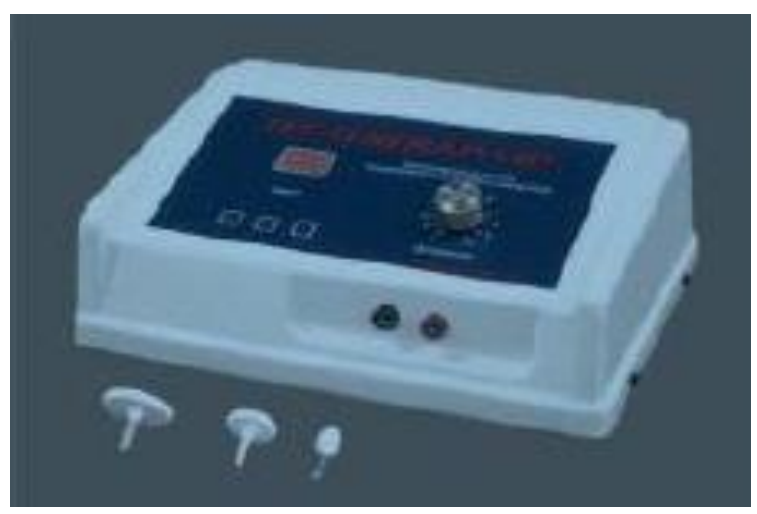

Fonte: Leal (2014, p. 21)

A radiofrequência tem trazido resultados significativos no tratamento da função vaginal. Ressaltaram os Gooddman et al. (2011), em um estudo com 33 mulheres que se submeteram a cirurgia vulvovaginal. Este estudo demonstrou que a cirurgia vulvovaginal e a RF tiveram resultados semelhantes em relação a função sexual.

O sexo tornou-se mais formidável as mulheres, com evidência no prazer sexual, que promoveu uma maior precaução voltada para o corpo feminino. Destacaram os autores Sekiguchi et al. (2013) e Lordêlo et al. (2016), em um estudo com aplicação de RF, concluíram que além de melhorar a aparência geral da genitália foi possível observar um aumento da lubrificação vaginal, sendo um ponto positivo.

A radiofrequência induz calor nos tecidos dérmicos (calor endógeno), aumentando a temperatura do tecido no sentido de alcançar uma temperatura local e proporcionando um aumento do fluxo sanguíneo na região. As participantes passaram a apresentar função sexual normal após o tratamento com a RF, tendo efeito positivo na função sexual (SANTOS, 2014).

Estudo realizado com uma amostra de 32 mulheres, sendo que 3 não completaram o tratamento, com idade entre 18 - 60 anos, com a finalidade de avaliar o efeito na função sexual, e foram submetidas ao tratamento da flacidez cutânea dos grandes lábios com RF. A 
partir dos resultados o autor Santos (2014), demonstrou a melhora da função sexual após o tratamento estético. Entretanto a RF aumenta a vascularização da região, mas não encontrou resultados significativo na lubrificação vaginal, sendo um aspecto negativo.

O efeito térmico da RF, faz com que ocorre a produção de novo colágeno pois ocorre uma contração nas suas fibras e como consequência a ativação dos fibroblastos com a formação da neocolagênese, fornecendo a essa pele mais firmeza e elasticidade. Leal (2014), em um estudo com 43 mulheres, porém 7 não finalizaram o tratamento. Os participantes do grupo estudo e do grupo controle tiveram satisfação com a RF em flacidez cutânea de grandes lábios vulvares, mostrando ser eficaz.

O Female Sexual Function Index (FSFI) é uma escala para avaliar a função sexual em mulheres, como confirma os autores Fistonic et al. (2016), em um estudo com 19 mulheres, sendo que 2 participantes desistiu, com idade entre 27 e 56 anos, apresentando flacidez vaginal, fizeram o procedimento com radiofrequência monopolar. Os resultados proporcionaram melhora na aparência vulvar, e melhora no funcionamento sexual medido pelo FSFI, contudo somente 1 não obteve alterações nos resultados.

Alterações na autoestima e na função sexual também podem ter influenciado no aumento da satisfação destas mulheres. Os autores Lordêlo et al. (2016) relataram um estudo com uma amostra composta de 43 mulheres, mas 7 participantes não concluíram o tratamento. Os resultados após o tratamento da RF nos grandes lábios genital foram satisfatório em relação a aparência da região genital, excitação sexual, e também houve um avanço no escore do FSFI.

A flacidez e decorrente de vários acontecimentos, como por exemplo o envelhecimento, parto vaginal, contudo o tecido não retorna a sua forma original perdendo a sua elasticidade, o que resulta em uma desagradável aparência da genitália feminina. Porém as mulheres buscam a cirurgia plástica da vagina (labioplastia) como opção de melhorar a vida sexual, autoestima e aparência da genitália (NOGUEIRA, 2017).

Contudo Nogueira (2017), aponta um método não invasivo e mais simples, a radiofrequência, sendo um método eficaz no tratamento da flacidez de vulva, que não apresenta complicações e nem riscos, como no caso de uma cirurgia. A partir do calor gerado pela radiofrequência (temperaturas entre 40 e $45^{\circ} \mathrm{C}$ ), ocorre algumas reações fisiológicas como aquecimento do tecido, vasodilatação local, estímulo à formação de novo colágeno (neocolagênese), aumento do fluxo sanguíneo, gerando assim a melhora da flacidez de pele.

264 Id on Line Rev. Mult. Psic. V.13, N. 45 SUPLEMENTO 1, p. 258-269, 2019 - ISSN 1981-1179 Edição eletrônica em http://idonline.emnuvens.com.br/id 
Segundo Qureshi et al. (2017), traz que as intervenções de NVR podem ser semelhante à neurotoxina ou preenchimento de tecidos moles no rejuvenescimento facial. A NVR com RF é importante na diminuição da flacidez da vagina e melhorar a relação sexual, sem a necessidade de cirurgia.

Incontinência urinária é a perda involuntária da urina pela uretra. O distúrbio é mais frequente no sexo feminino, já a frouxidão vaginal é o relaxamento e envelhecimento da região genitália, como corrobora os autores Lalji e Lozanova (2017), que realizaram um estudo com 27 amostras do sexo feminino com idade entre 28 e 66 anos, apresentaram incontinência urinária de esforço e frouxidão vaginal. Após o tratamento com o monopolar dispositivo de radiofrequência, os participantes teve a melhora significativa do vazamento urinário e da frouxidão vaginal. Nenhum dos participantes relatou insatisfeitos com o tratamento.

Apresenta-se procedimentos para o rejuvenescimento vaginal feminino não invasivo, pode-se destacar o laser de $\mathrm{CO}_{2}$ e a radiofrequência. $\mathrm{O}$ infravermelho causou aumento da espessura epitelial, vascularização e também melhorou a aparência estética e o estimulo no sexo feminino. Já a radiofrequência emitem efeitos eletromagnéticos ondas que aquecem os tecidos e não ocorre eventos adversos. O calor induz o colágeno a contração, promovendo que a elasticidade volte a sua forma original (HASHIM et al. 2018).

Demostra-se que o tratamento com a radiofrequência e lasers à base de $\mathrm{CO}_{2}$ não causa dor, não é invasivo, e aquece de forma bem suave o tecido da mucosa vaginal. Contudo em um estudo do autor Frota et al. (2018) o rejuvenescimento vulvovaginal foi testado pela RF e Lasers, com uma amostra de 23 mulheres com sintomas de flacidez vulvovaginal. Os resultados mostraram que todas as 23 mulheres tratadas apresentaram melhora na flacidez vaginal e contração das fibras de colágeno no canal vaginal, estabelecendo que o tratamento com RF é satisfatório e seguro, promovendo a melhora no prazer sexual.

Diante disso, é válido destacar que os autores, Gooddman et al. (2011), Sekiguchi et al. (2013), Santos (2014), Leal (2014), Fistonic et al. (2016), Lordêlo et al. (2016), Qureshi et al. (2017), Lalji et al. (2017), Nogueira (2017), Frota et al. (2018) e Hashim et al. (2018) destacam em seus estudos as contribuições da radiofrequência em pacientes com flacidez genital feminina, sendo uma alternativa eficaz e segura. Contudo, vale ressaltar a necessidade de mais estudos que abordam do uso desta terapia. 
Figura 2 - Antes e depois do tratamento com radiofrequência

Antes da RF / Depois da RF
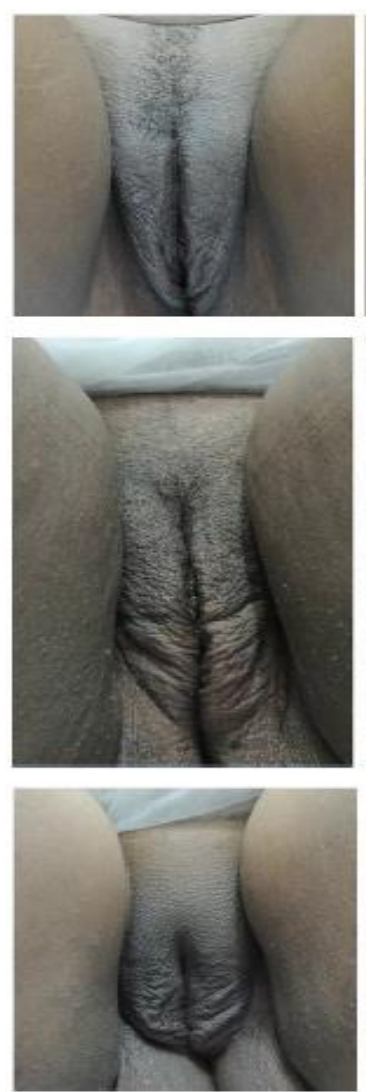
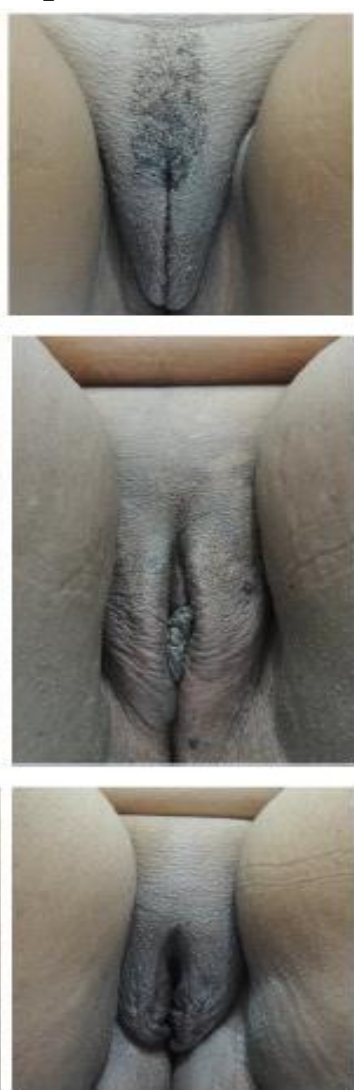

Antes da RF / Depois da RF
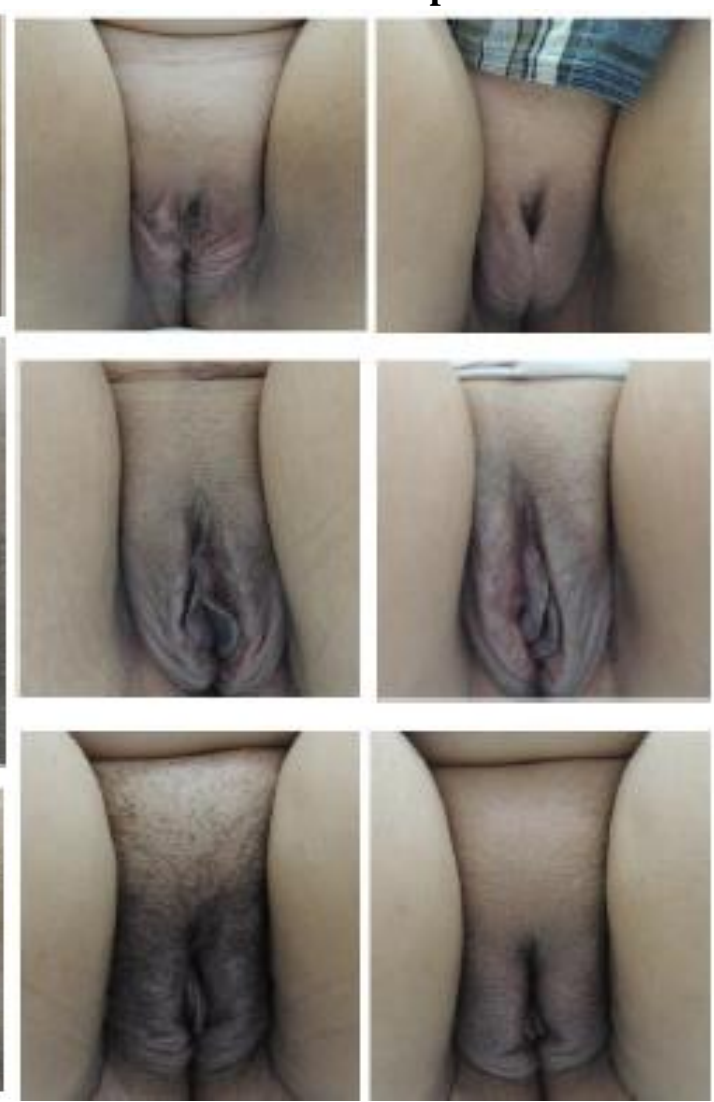

Fonte: Leal (2014, p. 38/39)

\section{Considerações Finais}

A flacidez genital dos grandes lábios é determinada pela ocorrência do envelhecimento e relaxamentos da região. Entretanto atinge homens e mulheres em todo o mundo, porém comprometendo mais as mulheres, sendo um problema que afeta a qualidade de vida das pessoas que são acometidas por esta situação.

Observou-se na presente revisão de literatura que a radiofrequência tem apresentado resultados positivos, como alternativa não invasiva para o tratamento da flacidez vulvar, sendo seguro, indolor e eficaz, melhorando a estética vaginal e disfunções sexuais relacionadas a flacidez em órgão genital feminino. 
A partir da análise dos resultados observou-se que a radiofrequência pode ser aplicada na região genital, apresentando baixos riscos de complicações, visto que existe a eficácia do tratamento, demostrando a elevação da melhora do desempenho sexual, na aparência e na autoestima.

Com base nos levantamentos de artigos sobre o tema, é comprovado a eficácia da radiofrequência como escolha não invasiva nas queixas de flacidez de grandes lábios, e tem apresentado resultados positivos melhorando o desempenho sexual, o rejuvenescimento genital e gerando a satisfação de mulheres com o tratamento.

Com relação as dificuldades para a realização do trabalho, a revisão de literatura foi uma etapa difícil do estudo, pois há uma escassez sobre este método de tratamento, estes foram os principais obstáculos encontrados. Sendo assim é imprescindível estudos posteriores sobre o tratamento com Radiofrequência.

\section{Referências}

BOCK, V., NORONHA, F.N; Estimulação da neocolanogênese através da radiofrequência, Revista eletrônica saúde e ciência, v.3, n.2, 2013.

FISTONIC, Ivan et al. Short Time Efficacy and Safety of Focused Monopolar Radiofrequency Device for Labial Laxity Improvement-Noninvasive Labia TissueTightening. A Prospective Cohort Study. Lasers in Surgery and Medicine, Croácia, v.48, n.3, p. $254-259$, jan. 2016.

FROTA, Taiane Carvalho et al. Tratamento com laser e radiofrequência da atrofia vulvovaginal: estudo bibliográfico. Revista Eletrônica Acervo Saúde. Universidade Federal do Amazonas (UFAM), Manaus-AM. p. 1 - 9, REAS/EJCH| Vol.Sup. 17 - 12/2018.

GOODMAN M, FASHLER S, MIKLOS JR., MOORE RD, BROTTO LA. The Sexual, Psychological, and Body Image Health of Women Undergoing Elective Vulvovaginal Plasticl Cosmetic Procedures: A Pilot Study. 28(4): 219-226. The American Journal of Cosmetic Surgery Vol. 28, No. 4, 2011.

HASHIM, Peter W. et al. Noninvasive Vaginal Rejuvenation. Cutis. 2018;102:243-246. VOL. 102 NO. 4 I OCTOBER 2018.

JUNQUEIRA LC, CARNEIRO J: Aparelho Reprodutor Feminino. In: Junqueira LC, Carneiro J (autores), Histologia Básica. 8. ed., Rio de Janeiro, Guanabara Koogan, 1995, 367388 . 
LALJI, Shelena; LOZANOVA, Paula. Evaluation of the safety and efficacy of a monopolar nonablative radiofrequency device for the improvement of vulvo-vaginal laxity and urinary incontinence. Accepted: 29 March 2017. J Cosmet Dermatol. 2017;1-5.

LEAL; Mariana Robatto Dantas. Radiofrequência em Região Genital Feminina: um Ensaio Clínico Randomizado. 2014. Dissertação de Mestrado - Escola de Medicina e Saúde PublicaBAHIANA. Salvador-Bahia, 2014.

LORDÊLO, Patrícia et al. Radiofrequency in female external genital cosmetics and sexual function: a randomized clinical trial. Published online: 26 April 2016. The International Urogynecological Association 2016.

MATTAR, F. N. Pesquisa de marketing. 3.ed. São Paulo: Atlas, 2001.

MORETTI, Isabella. Metodologia de Pesquisa do TCC: conheça os tipos e veja como definir. 26/07/2018. Disponível em: < https://viacarreira.com/metodologia-de-pesquisa-do-tcc110040/>. Acesso em: 10 jan. 2019.

NOGUEIRA, Madímena Santos. Aplicação da Radiofrequência em Órgão Genital Feminino: Um Procedimento não Invasivo no Tratamento da Flacidez de Pele Vulvar. 2017. Trabalho de Conclusão de Curso, UNIME-Lauro de Freitas, 2017.

QURESHI, Ali A. et al. Nonsurgical Vulvovaginal Rejuvenation With Radiofrequency and Laser Devices: A Literature Review and Comprehensive Update for Aesthetic Surgeons. Aesthetic Surgery Journal. Editorial Decision date: July 4, 2017.

SANTOS; Juliana Menezes. Função Sexual Após o Tratamento com a Radiofrequência em Região Genital Feminina: Ensaio Clínico Randomizado. 2014. DISSERTAÇÃO DE MESTRADO. Escola Bahiana de Medicina e Saúde Pública -SALVADOR-BAHIA, BRASIL, 2014.

SEKIGUCHI Y, UTSUGISAWA Y, AZEKOSI Y, KINJO M, SONG M, KUBOTA Y, KINGBERG SA, KRYCHMAN ML (2013) Laxity of the vaginal introitus after childbirth: nonsurgical outpatient procedure for vaginal tissue restoration and improved sexual satisfaction using low-energy radiofrequency thermal therapy. J Womens Health 22(9):77581.

VERGARA, Sylvia C. Projetos e relatórios de pesquisa em administração. 3.ed. Rio de Janeiro: Atlas, 2000.

VIEIRA, Giovanna de Simone Kaadi. Importância da Radiofrequência em Tratamentos Estéticos: Revisão da Literatura. Trabalho Conclusão de Curso. PONTIFÍCIA UNIVERSIDADE CATÓLICA DE GOIÁS - GOIÂNIA-GO, 2016.

ZEMUNER, E.; GUIDI, R. M. Radiofrequência para tratamentos estéticos: mitos e verdades, $6^{\circ}$ Congresso Científico Internacional de Estética e Cosmetologia \pm 9 CCIEC, São Paulo, 2011.

268 Id on Line Rev. Mult. Psic. V.13, N. 45 SUPLEMENTO 1, p. 258-269, 2019 - ISSN 1981-1179 Edição eletrônica em http://idonline.emnuvens.com.br/id 
ZIKMUND, W. G. Business research methods. 5.ed. Fort Worth, TX: Dryden, 2000.

\section{Como citar este artigo (Formato ABNT):}

LEAL, Talita Pereira; SANTOS, Juliana Amorim Borba. Contribuições da Radiofrequência em flacidez genital feminina: Uma Revisão da Literatura. Id on Line Rev.Mult. Psic., 2019, vol.13, n.45 SUPLEMENTO 1, p. 258-269. ISSN: 1981-1179.

Recebido: 13/05/2019

Aceito 19/05/2019 